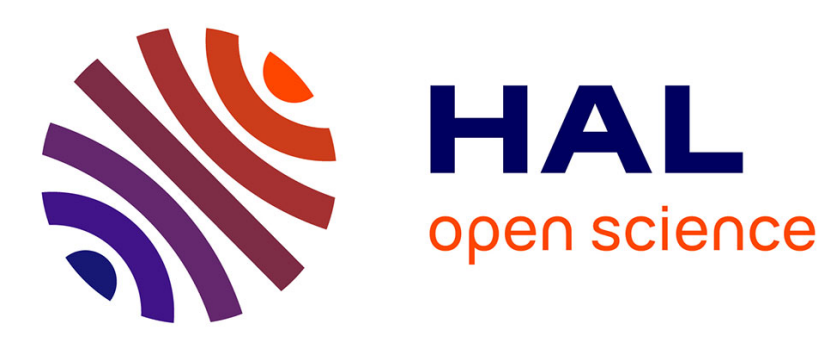

\title{
MHD modeling of the Heliosphere: a critical evaluation of different models
}

R. Ratkiewicz

\section{To cite this version:}

R. Ratkiewicz. MHD modeling of the Heliosphere: a critical evaluation of different models. Astrophysics and Space Sciences Transactions, 2006, 2 (1), pp.11-17. hal-00297475

\section{HAL Id: hal-00297475 \\ https://hal.science/hal-00297475}

Submitted on 3 Feb 2006

HAL is a multi-disciplinary open access archive for the deposit and dissemination of scientific research documents, whether they are published or not. The documents may come from teaching and research institutions in France or abroad, or from public or private research centers.
L'archive ouverte pluridisciplinaire HAL, est destinée au dépôt et à la diffusion de documents scientifiques de niveau recherche, publiés ou non, émanant des établissements d'enseignement et de recherche français ou étrangers, des laboratoires publics ou privés. 


\title{
MHD modeling of the Heliosphere: a critical evaluation of different models
}

\author{
R. Ratkiewicz \\ Space Research Center PAS, Bartycka 18A, 00-716 Warsaw, Poland
}

Received: 6 October 2005 - Revised: 22 November 2005 - Accepted: 28 November 2005 - Published: 2 February 2006

\begin{abstract}
The very complicated problem of the solar windinterstellar medium interaction requires a very sophisticated numerical approach. To achieve such a goal first we need to understand very deeply the results obtained up-to-now. In this paper we gather the results from four different MHD models of the heliospheric interfaces. The comparison of the results invokes several questions. These questions should be addressed before we proceed with the next steps in the MHD modeling of the heliospheric interfaces. Is a jet created between the termination shock and the heliopause (Opher et al., 2003, 2004)? What is the physical meaning of the V-shape of termination shock and heliopause (Washimi and Tanaka, 2001, 2004)? Whether a similar looking result is either due to bending of the heliospheric current sheet as in the model by Pogorelov (2004), Pogorelov et al. (2004) or due to numerical "reconnection" as in the model by Ratkiewicz et al. (2004, 2005)? The purpose of this paper is to open a wider discussion to try to answer these questions.
\end{abstract}

\section{Introduction}

It is well recognized that the problem of the solar wind (SW) - local interstellar medium (LISM) interaction is very complicated. The basis for this interaction is the mutual interaction of the solar wind plasma and the ionized component of the interstellar medium. The gasdynamic treatment of problem gives the heliospheric interfaces: the termination shock (TS), the heliopause (HP), and possibly the bow shock (BS). The region between TS and HP is the heliosheath (HS).

Deep interplanetary missions (Pioneer, Voyager 1 and 2) as well as SOHO, Ulysses and HST provide data on the solar wind, the local interstellar medium, and the heliospheric interfaces. It is widely accepted that Voyager 1 has crossed the termination shock in the solar wind at the distance $94 \mathrm{AU}$ (Stone et al., 2005), and now is surfing in the heliosheath. The Interstellar Boundary Explorer (IBEX), a new genera-

Correspondence to: R. Ratkiewicz (roma@cbk.waw.pl) tion deep space mission with multiple instruments on board, is scheduled to be launched in 2008. It will make the first map of the boundary between the heliosphere and interstellar space. Therefore in the next few years we may have new and powerful tools to investigate the heliospheric boundary. To make proper use of the images of the interstellar boundaries beyond our solar system, we should be prepared to confront our theoretical models with images and data. The models should encompass the interaction of both plasmas in the presence of interplanetary and interstellar magnetic fields, and also galactic cosmic rays (GCR's); interactions of plasmas in different regions of the interface with the LISM neutral component, and all the consequences resulting from these interactions, due to pick-up ions (PUI's), anomalous cosmic rays (ACR's), energetic neutral atoms (ENA's); possible time-dependent phenomena, latitudinal dependence of SW, etc. The problem needs to be solved self-consistently using a 3-D time-dependent multicomponent MHD code that employs an adequate 3-D kinetic model for the neutral atoms distribution. Many different approaches have been done to investigate different aspects of the SW-LISM interaction, but no model is entirely satisfactory.

The most developed gasdynamic model has been worked out by Fahr et al. (2000). The 2-D model includes the interaction of SW and LISM protons, pick-up ions, neutral hydrogen atoms, anomalous cosmic rays, and galactic cosmic rays within a self-consistent 5 -fluid approach. The first attempt to include into numerical models the magnetic fields was made by Fujimoto and Matsuda (1991). Since then several modelers have made a big effort to model magnetohydrodynamically the heliospheric interfaces including the interstellar magnetic field (ISMF), and/or the interplanetary magnetic field (IPMF), and/or neutral (N) components of LISM (Baranov and Zaitsev, 1995; Washimi and Tanaka, 1996; Pogorelov and Semenov, 1997; Linde et al., 1998; Pogorelov and Matsuda, 1998; Ratkiewicz et al., 1998; McNutt et al., 1999; Washimi and Tanaka, 1999; Aleksashov et al., 2000; Ratkiewicz et al., 2000; Washimi and Tanaka, 2001; Ratkiewicz et al., 2002; Ratkiewicz and Ben-Jaffel, 
Table 1. The conditions at the boundaries in the solar wind and the interstellar medium

\begin{tabular}{|c|c|c|c|c|c|c|c|c|c|c|c|c|c|}
\hline $\begin{array}{l}\text { Model } \\
\text { Number }\end{array}$ & $\begin{array}{c}R_{\text {in }} \\
\mathrm{AU}\end{array}$ & $\begin{array}{l}V_{s w} \\
\mathrm{~cm} / \mathrm{s}\end{array}$ & $M_{s w}$ & $\begin{array}{c}T_{s w} \\
10^{3} \mathrm{~K}\end{array}$ & $\begin{array}{c}n_{s w} \\
\mathrm{~cm}^{-3}\end{array}$ & $\begin{array}{c}B_{s w} \\
\mu G\end{array}$ & $\begin{array}{c}R_{\text {out }} \\
\mathrm{AU}\end{array}$ & $\begin{array}{l}V_{i s} \\
\mathrm{~cm} / \mathrm{s}\end{array}$ & $M_{i s}$ & $\begin{array}{c}T_{i s} \\
10^{3} \mathrm{~K}\end{array}$ & $\begin{array}{c}n_{i s} \\
\mathrm{~cm}^{-3}\end{array}$ & $\begin{array}{l}B_{i s} \\
\mu G\end{array}$ & $\begin{array}{c}n_{H} \\
\mathrm{~cm}^{-3}\end{array}$ \\
\hline $1: O$ & 30 & 450 & & 1.6 & 0.0078 & 2.0 & $R_{1}$ & 25.0 & & 10 & 0.07 & 0 & 0.0 \\
\hline $2: W$ & 50 & 400 & & 10.0 & 0.0020 & $0.84 *$ & $R_{2}$ & 22.5 & & 10 & 0.09 & 1.2 & 0.0 \\
\hline $3: P$ & 1 & 450 & 10 & & 7.0 & $37.5^{* *}$ & $R_{3}$ & 25.0 & 2.0 & & 0.07 & var & 0.0 \\
\hline $4: R$ & 30 & 400 & 10 & & 0.0089 & 2.0 & $R_{4}$ & 26.0 & 1.87 & & 0.043 & var & 0.2 \\
\hline $\begin{array}{l}R_{1}=345 \\
\text { *) } B_{\phi}=0\end{array}$ & $\begin{array}{l}U \times \\
\text { in } t+1\end{array}$ & $0 \mathrm{AL}$ & 4500 & J, & $\begin{array}{l}R_{2}=950 \\
\beta_{\phi}=0.84 \mathrm{~s}\end{array}$ & & \multicolumn{2}{|c|}{$\begin{array}{l}R_{3}=\text { not given, } \\
\text { **) } B_{r}\end{array}$} & \multicolumn{3}{|c|}{$R_{4}=15000 \mathrm{AU}$} & & \\
\hline
\end{tabular}

2002; Ratkiewicz and Webb, 2002; Opher et al., 2003; Ratkiewicz and McKenzie, 2003; Florinski et al., 2004; McNutt, 2004; Opher et al., 2004; Pogorelov, 2004; Pogorelov et al., 2004; Ratkiewicz and Webb, 2004; Ratkiewicz et al., 2004; Washimi and Tanaka, 2004; Ratkiewicz et al., 2005).

Although none of these models is perfect, each of them gives new information or pays attention to some special aspects of the complicated MHD interaction (with or without the neutral particles), such as asymmetries introduced by interplanetary or interstellar magnetic fields, obliqueness of the bow shock and heliopause, heliospheric currents, especially the heliospheric current sheet (HCS), the role of the neutral particles and the solar cycle, jets, V-shape, bending HCS, numerical "reconnection," and instabilities. As mentioned in Ratkiewicz et al. (2005) the results are, in general, in a good agreement. The heliospheric interfaces obtained from different models look similar. However, they differ in details or in the interpretation of these details. It is thus important to re-assess the physical validity of the different models. In the next section, as examples, four models, called 1:O (Opher), 2:W (Washimi), 3:P (Pogorelov), and 4:R (Ratkiewicz) model, respectively, are described and discussed. Is a jet (model 1:O, Opher et al., 2003, 2004) created between the termination shock and the heliopause? What is the physical meaning of the V-shape of TS and HP in the model 2:W (Washimi and Tanaka, 2001, 2004)? Similarly, is there bending of the heliospheric current sheet as in the model 3:P (Pogorelov, 2004; Pogorelov et al., 2004), or numerical "reconnection" as claimed in model 4:R (Ratkiewicz et al., 2004, 2005)? The purpose of this paper is to initiate a wider discussion on the physical meaning of these phenomena.

\section{Four models}

2.1 MHD equations and conditions at the boundaries

The set of MHD equations may be written in the form:

$$
\frac{\partial \mathbf{U}}{\partial t}+\nabla \cdot \overline{\mathbf{F}}=\mathbf{Q}+\mathbf{S}
$$

where $\mathbf{U}, \mathbf{Q}$, and $\mathbf{S}$ are column vectors, and $\overline{\mathbf{F}}$ is a flux tensor. $\mathbf{U}$, and $\overline{\mathbf{F}}$ are defined as:

$$
\mathbf{U}=\left|\begin{array}{c}
\rho \\
\rho \mathbf{u} \\
\mathbf{B} \\
\rho E
\end{array}\right| \quad \overline{\mathbf{F}}=\left|\begin{array}{c}
\rho \mathbf{u} \\
\rho \mathbf{u u}+\mathbf{I}\left(p+\frac{\mathbf{B} \cdot \mathbf{B}}{8 \pi}\right)-\frac{\mathbf{B B}}{4 \pi} \\
\mathbf{u B}-\mathbf{B u} \\
\left.\rho H \mathbf{u}-\frac{\mathbf{B}(\mathbf{u} \cdot \mathbf{B}}{4 \pi}\right)
\end{array}\right|
$$

In the 4:R model, the RHS (1) has two source terms: a source term $\mathbf{S}$ describing charge exchange with the constant flux of hydrogen atoms, and a source term $\mathbf{Q}$ responsible for numerically enforcing $\nabla \cdot \mathbf{B}=0$. In the $1: \mathrm{O}, 2: \mathrm{W}$, and 3:P models $\mathbf{S}=0$, since the neutral particles are neglected. $\mathbf{Q}$ and $\mathbf{S}$ are given by

$$
\begin{gathered}
\mathbf{Q}=-\left|\begin{array}{c}
\frac{0}{\mathbf{B}} \\
\frac{4 \pi}{\mathbf{u}} \\
\mathbf{u} \cdot \frac{\mathbf{B}}{4 \pi}
\end{array}\right| \nabla \cdot \mathbf{B} \\
\mathbf{S}=\rho \nu_{c}\left|\begin{array}{c}
0 \\
\mathbf{V}_{\mathbf{H}}-\mathbf{u} \\
\mathbf{0} \\
\frac{1}{2} V_{H}{ }^{2}+\frac{3 k_{B} T_{H}}{2 m_{H}}-\frac{1}{2} u^{2}-\frac{k_{B} T}{(\gamma-1) m_{H}}
\end{array}\right|
\end{gathered}
$$

Here, $\rho$ is the ion mass density, $p=2 n k_{B} T$ is the pressure, $n$ is the ion number density, $T$ and $T_{H}\left(T_{H}=\right.$ const $)$ are ion and $\mathbf{H}$-atom temperatures, and $\mathbf{u}$ and $\mathbf{V}_{\mathbf{H}}\left(\mathbf{V}_{\mathbf{H}}=\right.$ const $)$ are the ion and $\mathrm{H}$-atom velocity vectors, respectively; $\mathbf{B}$ is the magnetic field vector, $E=\frac{1}{\gamma-1} \frac{p}{\rho}+\frac{\mathbf{u} \cdot \mathbf{u}}{2}+\frac{\mathbf{B} \cdot \mathbf{B}}{8 \pi \rho}$ is the total energy per unit mass, $H=\frac{\gamma}{\gamma-1} \frac{p}{\rho}+\frac{\mathbf{u} \cdot \mathbf{u}}{2}+\frac{\mathbf{B} \cdot \mathbf{B}}{4 \pi \rho}$, $\gamma$ is the ratio of specific heats. $\quad I$ is the $3 \times 3$ identity matrix. The charge exchange collision frequency is $\nu_{c}=n_{H} \sigma u_{*}$, where $n_{H} \quad\left(n_{H}=\right.$ const $)$ is the H-atom number density, $\sigma$ is the charge exchange cross-section, and $u_{*}=\left(\left(\mathbf{u}-\mathbf{V}_{\mathbf{H}}\right)^{2}+128 k_{B}\left(T+T_{H}\right) /\left(9 \pi m_{H}\right)\right)^{1 / 2}$ is the effective average relative speed of protons and H-atoms, assuming a Maxwellian spread of velocities both for protons and H-atoms. The flows are taken to be adiabatic with $\gamma=5 / 3$. 
Table 2. Four Simulation Models

\begin{tabular}{|c|c|c|c|c|}
\hline Model & ISMF & $\mathrm{N}$ & IPMF & Features \\
\hline 1: $\mathrm{O}$ & - & - & $\mathrm{P}$ & jet \\
\hline 2: W & $\alpha=90^{\circ}$ & - & $\mathrm{P}$ & $B_{\phi}-\mathrm{V}$ shape \\
\hline 3: P & $\begin{array}{l}\alpha=0,45,90^{\circ} \\
\theta=0,60^{\circ}\end{array}$ & - & $\mathrm{P}$ & HCS bending \\
\hline 4: R & $\begin{array}{l}0 \leq \alpha \leq 90^{\circ} \\
\theta=0,60^{\circ}\end{array}$ & $\mathrm{CF}$ & $\mathrm{P}$ & $\begin{array}{l}\text { numerical } \\
\text { "reconnection" }\end{array}$ \\
\hline \multicolumn{4}{|c|}{$\begin{array}{l}\text { N-neutrals } \quad \text { P-Parker model } \\
\alpha \text { - angle between } V_{i s} \text { and } B_{i s} \\
\theta \text { - deviation angle from ecliptic plane }\end{array}$} & CF-constant flux \\
\hline
\end{tabular}

The set of differential equations to be solved requires conditions at the boundaries. Those at the computational boundaries play a very important role. They are given in Table 1 . We use the same notation as in the original papers by Opher et al. (2004); Washimi and Tanaka (2004); Pogorelov et al. (2004), and Ratkiewicz et al. (2004). At this point we want to stress that the comparison of the results of the above models is difficult because of the different boundary conditions used. The main characteristic features for each of the four models are listed in Table 2.

\subsection{Model 1: Opher model: IPMF, no ISMF, no N}

The main result of this model is a jet. According to Opher et al. (2003, 2004), "the jet-sheet structure forms in the region of minimum of magnetic pressure. The jet extends for $150 \mathrm{AU}$ beyond the TS, almost touching the BS. There is a back flow swept aside by the jet. Downstream of the shock, where the flow decelerates, conservation of magnetic flux outside of the equatorial plane causes the field to increase its magnitude further, while in the current sheet there is no such effect. As a result, the increased magnetic field above and below the ecliptic planes effectively pinches the sheet just beyond the TS, causing the stream lines in the subsonic regions to converge slightly.... The converging flow lines near the equatorial plane create a de Laval nozzle". According to the authors this explains the formation of the jet. However, they admit that "the jet is unstable". This could be an interesting feature of the boundary between the solar wind and the interstellar medium, but is probably unrealistic. If we agree that there are very important effects of the interstellar magnetic field and neutral particles, studies not taking them into account are questionable. Also we are concerned about the shape of the termination shock shown in Fig. 1a,b (p. 577 in Opher et al., 2004). The shape of the TS in these figures does not reflect the Mach disc, especially characteristic for the flow without the neutral particles.

\subsection{Model 2: Washimi model: IPMF, ISMF, no N}

The results obtained by Washimi and Tanaka (2001, 2004) seem to contradict the above results. In this case both interplanetary and interstellar magnetic fields are present in the model. Where a jet is created in the 1:O model, the V- shape gutter has been obtained in the 2:W model (see Figs. 3 and 4 in Washimi and Tanaka, 2004). In the process of the solar wind-interstellar plasma interaction the nose-cone type HP (steady-state) for initially No-Sheet Condition is obtained. "After the inner boundary is switched to the Sheet Condition from the No-Sheet Condition ... a neutral sheet is formed not only in the interplanetary space but also in the heliosheath region. At the same time, lower density regions... appear... near the neutral sheet where the intensity of $\mathbf{B}_{\phi}$ should be strong due to $\sin \theta$ dependence. These low density regions are found to enlarge with time because the solar wind plasma supply from the inner side is insufficient, and finally these regions in both hemispheres are found to connect with the interstellar medium, which results in the invasion of it at low latitudes. Thus a V-shaped gutter is formed." The authors say that "the reason why such a V-shaped structure was not obtained in previous MHD studies (e.g. Linde et al., 1998; Opher et al., 2003) may be due to different inner boundary conditions."

\subsection{Model 3: Pogorelov model: IPMF, ISMF, no N}

One of the most characteristic features of this model is the bending of the heliospheric current sheet (HCS). The results concerning the HCS are summarized in the two following quotations. According to the statement in Pogorelov (2004): "Figs. $3 \mathrm{a}$ and $3 \mathrm{~b}$ show the meridional-plane distributions of density logarithm and magnetic field magnitude $\mathbf{B}_{\text {tot }}$, respectively, assuming $\mathbf{B}_{\infty} \approx 1.5 \mu \mathrm{G}$. Clearly, the HCS, as a layer with small $\mathbf{B}_{\text {tot }}$ experiences substantial bending to the southern hemisphere. This is opposite to its bending direction in the switch-on regime. The HCS exhibits periodic oscillations, as well as causing the bump on the surface of the heliopause." In the paper Pogorelov et al. (2004) "a particularly important result is the demonstrated bending (and possible rotation) of the HCS after it crosses the TS. It is important to note that solutions of ideal MHD problems might have little physical meaning. For example, if $\mathbf{B}_{\infty} \perp \mathbf{V}_{\infty}$ and $\mathbf{B}_{\infty} \perp O x$, the solution must be symmetric with respect to the ecliptic plane. In fact, as seen in Fig. 6, the solution becomes asymmetric and the bending of the HCS acts to prevent reconnection in the lower hemisphere. In principle, numerical viscosity and resistivity do not allow us to perform a very detailed investigation of the HCS. For example, for certain orientations of the ISMF, solutions exhibit unsteadiness. However, the global behavior of HCS is quite well resolved, and its orientation may be useful in providing some information about the LIC."

\subsection{Model 4: Ratkiewicz model: IPMF, ISMF, $\mathrm{N}$ const.}

The interpretation of the phenomenon called the bending HCS is different in Ratkiewicz et al. (2004, 2005). The 4:R model shows the numerical "reconnection" (Linde et al., 1998) rather than bending of the current sheet. The presence of a code-dependent numerical diffusivity is an intrinsic property of numerical MHD codes. Even if the ideal 

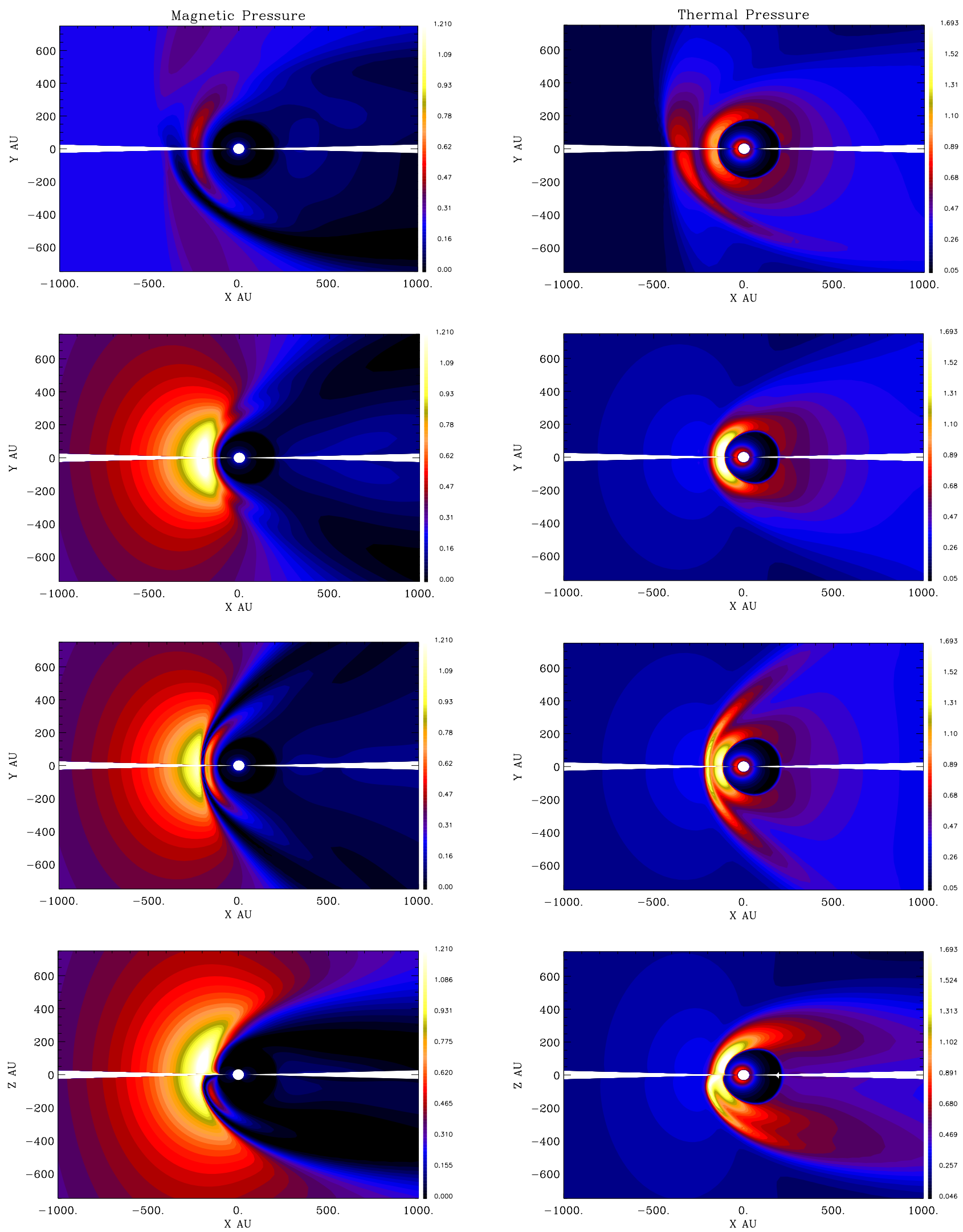

Fig. 1. Magnetic pres (left) and thermal pres (right): 1st row: $\boldsymbol{B}_{\text {is }} \| \boldsymbol{V}_{\text {is }}$ with reconnection in x-y, 2nd row: $\boldsymbol{B}_{\text {is }} \perp \boldsymbol{V}_{\text {is }}$ with no reconnection in x-y, 3rd row the same as 2 nd but with reconnection, 4th row: $\boldsymbol{B}_{i s} \perp \boldsymbol{V}_{i s}$, x-z plane with no reconnection at northern, and with reconnection at southern hemisphere 

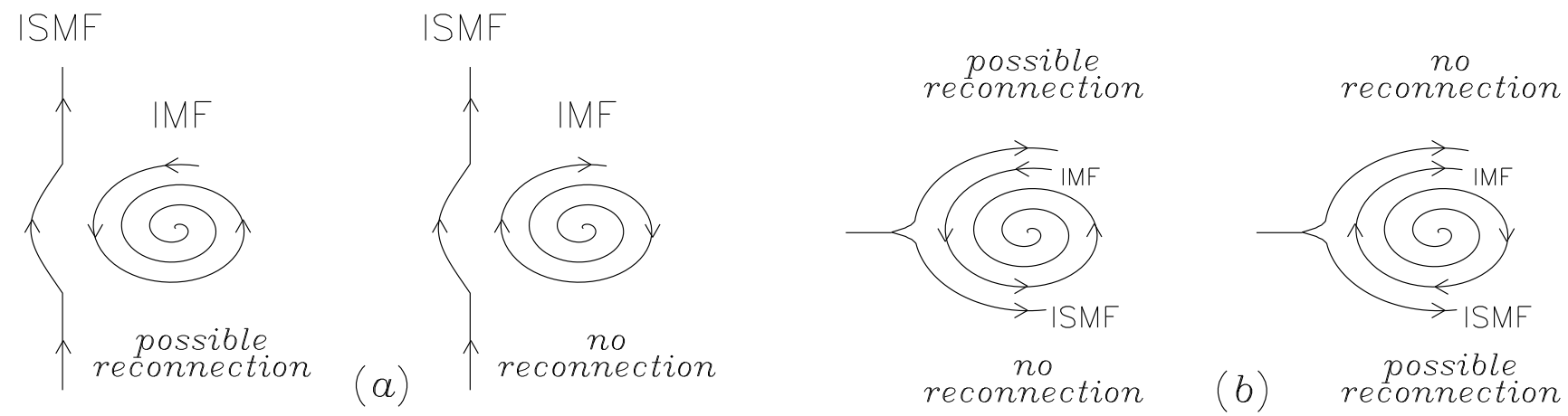

Fig. 2. Schematic of possible reconnection for (a) $\boldsymbol{B}_{i s} \perp \boldsymbol{V}_{i s}$, (b) $\boldsymbol{B}_{i s} \| \boldsymbol{V}_{i s}$, and interplanetary magnetic field spiraling toward (left (a) and (b)) the Sun, and away from the Sun (right (a) and (b))

MHD set of equations is solved, the numerical diffusion of the magnetic field may lead to numerical "reconnection", and changes of the magnetic field topology. The numerical "reconnection" at the heliopause indicates the possibility of new asymmetries of the heliospheric interface besides that caused by the ISMF (Ratkiewicz et al., 2004). However, it does not give the precise reconnection rate and location. Such a study has a meaning for the future investigation of the physical reconnection at the heliopause, and asymmetries caused by it. For this purpose one must solve a set of resistive 3-D MHD equations including the resistive terms involving $\eta \boldsymbol{J}(\boldsymbol{J}=\nabla \times \boldsymbol{B}$ and $\eta$ is resistivity) in the induction and energy equations.

\section{Discussion}

In order to understand what is true or false in numerical simulations, consider the set (1) of ideal MHD equations. One should note that the magnetic field in all equations is represented by terms having the dimension $B^{2}$. This remark should be taken into account in numerical calculations of the heliospheric interfaces. Although the interplanetary magnetic field changes polarity across the ecliptic plane, the sign of the magnetic field has no meaning for the terms with the magnetic field in the set (1) of ideal MHD equations. If it would be possible to separate ideally the heliosphere from the interstellar region (which means if the heliopause would be an ideal separatrix), it would mean practically for numerical simulations that the sign should not be changed. Note however, that the global topology of the magnetic field depends on the sign of $\boldsymbol{B}$.

In all above cited papers, the modelers have changed the polarity in the calculations. We suggest to repeat the calculations without a change the sign for IPMF, however with inclusion of the correct model of the HCS. It would be the first step allowing to avoid the numerical "reconnection" northsouth, which appears in Fig. 1, the last row (reproduced from Fig. 4 in Ratkiewicz et al., 2004), and see the behaviour of the HCS.
It is more difficult to avoid numerical "reconnection" at the heliopause. As the example let's refer to cases for two inclination angles $\alpha=0$ and $90^{\circ}$ of the ISMF, illustrated in Fig. 1 (1st and 3rd rows). The presented results are obtained from our model with $\mathbf{B}_{\text {is }}$ and $\mathbf{V}_{\text {is }}$ contained in the $\mathrm{x}-\mathrm{y}$ (ecliptic) plane, the LISM velocity vector in the positive $\mathrm{x}$-direction, and the $\mathrm{z}$-axis parallel to the solar rotation axis. The sign of the interplanetary magnetic field (IPMF is spiraling toward the Sun) is not changed and the HCS is not modelled. Figure 1 (1st and 3rd rows) displays the magnetic field and thermal pressure in the plane parallel to the $x-y$ (ecliptic) plane. For both directions (1st and 3rd rows) of the ISMF, the configuration of both magnetic fields is in favour of the numerical "reconnection". For $\alpha=90^{\circ}$ the perfect symmetry is saved (Fig. 1, the 3rd row). As the results of our calculations show for decreasing inclination angle the asymmetry caused by numerical "reconnection" increases, and is largest for $\alpha=0^{\circ}$ (compare Fig. 1, the 1st row). The numerical "reconnection" causes the new asymmetry for $\alpha=0^{\circ}$. Note that if the IPMF is spiraling from the Sun for the same ISMF inclination angles as above, it does not cause the numerical "reconnection" for quasi-perpendicular magnetic fields (compare Fig. 1, the 2nd row), but for quasi-parallel magnetic fields always does (as explained in Fig. 2b, reproduced from Fig. 1 b in Ratkiewicz et al., 2004). So, in order to avoid it one should model the interaction of ISMF and IPMF in such a way, to exclude different signs of the magnetic fields on the both sides of the heliopause.

\section{Conclusions}

Summarizing: jet or V-shape gutter? Bending HCS or numerical "reconnection" or maybe both? Which process is the one occurring in nature?

In order to answer the above questions, modellers should build numerical codes, which exclude physically unreasonable results. We should use the same boundary conditions. It is very crucial to choose the proper solar wind parameters. Since there are at least two modes of the solar wind flow, the slow and fast wind (Axford and McKenzie, 1997), we pro- 
Table 3. The propose conditions at the boundaries in the solar wind and the interstellar medium

\begin{tabular}{|c|c|c|c|c|c|c|c|c|c|c|c|c|}
\hline a) & $\begin{array}{l}r_{i n} \\
\mathrm{AU}\end{array}$ & $\begin{array}{l}V_{s w} \\
\mathrm{~cm} / \mathrm{s}\end{array}$ & $M_{s w}$ & $\begin{array}{l}n_{s w} \\
\mathrm{~cm}^{-3}\end{array}$ & $\begin{array}{l}B_{s w} \\
\mu G\end{array}$ & b) & $\begin{array}{l}R_{\text {out }} \\
\mathrm{AU}\end{array}$ & $\begin{array}{l}V_{i s} \\
\mathrm{~cm} / \mathrm{s}\end{array}$ & $\begin{array}{l}T_{i s} \\
10^{3} \mathrm{~K}\end{array}$ & $\begin{array}{l}n_{i s} \\
\mathrm{~cm}^{-3}\end{array}$ & $\begin{array}{l}B_{i s} \\
\mu G\end{array}$ & $\begin{array}{l}n_{H} \\
\mathrm{~cm}^{-3}\end{array}$ \\
\hline & $r_{i n}$ & 400 & 10 & $10 / r_{i n}^{2}$ & $2.0^{*}$ & & $R_{\text {out }}$ & 26 & 7 & 0.1 & $1.5-3.0$ & 0.22 \\
\hline & $\begin{array}{l}r_{i n} \\
*)-\end{array}$ & $\begin{array}{l}30-\mathrm{t} \\
\text { the ec }\end{array}$ & $\begin{array}{l}\text { inner } \\
\text { intic pla }\end{array}$ & oundary & & & \multicolumn{6}{|c|}{$R_{\text {out }}$ - the outer boundary according to the model } \\
\hline
\end{tabular}

pose to use the slow solar wind properties (Table 3a), which are commonly accepted. For the LISM we propose to use data (Table 3b) given by Frisch (2004).

We propose to calculate in each model two cases: without and, if possible, with the neutral particles (that is why the number density of $\mathrm{H}$-atom is also given in the last column in Table 3). Only then we will be able to compare our results.

Acknowledgements. The author acknowledges a support from the DFG-PAS cooperation project 436 POL 113/90/0-3, also a support in part from: the KBN Grant No.1P03D 009 27, CNRS/PAN Program 18249 and ASTRO-PF Program.

Edited by: H.-J. Fahr

Reviewed by: two referees

\section{References}

Aleksashov, D. B., Baranov, V. B., Barsky, E. V., and Myasnikov, A. V.: An Axisymmetric Magnetohydrodynamic Model for the Interaction of the Solar Wind with the Local Interstellar Medium, Astron. Letters, 26, 743-749, 2000.

Axford, W. I. and McKenzie, J. F.: The Solar Wind, in: Cosmic Winds and the Heliosphere, edited by Jokipii, J. R., Sonett, C.P., and Giampapa, M. S., Univ. of Ariz. Press, 31-66, 1997.

Baranov, V. B. and Zaitsev, N. A.: On the problem of the solar wind interaction with magnetized interstellar plasma, Astron. Astophys., 304, 631-637, 1995.

Fahr, H. J., Kausch, T., and Scherer, K.: A 5-fluid hydrodynamic approach to model the solar system-interstellar medium interaction, Astron. Astrophys., 357, 268-282, 2000.

Florinski, V., Pogorelov, N. V., Zank, G. P., Wood, B. E., and Cox, D. P.: On the Possibility of a Strong Magnetic Field in the Local Interstellar Medium, Astrophys. J., 604, 700-706, 2004.

Frisch, P.: The Solar Galactic Environment, in: AIP Conference Proceedings 719: Physics of the outer Heliosphere: 3rd International IGPP Conference, edited by Florinski, V., Pogorelov, N. V., and Zank, G. P., 404-411, 2004.

Fujimoto, Y. and Matsuda, T.: Interaction between the Solar Wind and Magnetized Local Interstellar Medium, KUGD91-2, Dep. Aeronaut. Eng., Kyoto University, Kyoto, Japan, 1991.

Linde, T., Gombosi, T., Roe, P., Powell, K., and DeZeeuw, D.: Heliosphere in the magnetized local interstellar medium - Results of a three-dimensional MHD simulation, J. Geophys. Res., 103, 1889-1904, 1998.

McNutt, R. L., Jr.: Modeling Charge Exchange in the Solar Wind/VLISM Interaction, in: AIP Conference Proceedings 719: Physics of the outer Heliosphere: 3rd International IGPP Conference, edited by Florinski, V., Pogorelov, N. V., and Zank, G. P., 111-116, 2004.
McNutt, R. L., Jr., Lyon, J., Goodrich, C. C., and Wiltberger, M.: 3D MHD Simulations of the Heliosphere-VLISM Interaction, in: AIP Conference Proceedings 471: Solar Wind Nine: 9th International Solar Wind Conference, edited by Habbal, S. R., Esser, R. Hollveg, J. V., and Isenberg, P. A., 823-826, 1999.

Opher, M., Liewer, P. C., Gombosi, T. I., Manchester, W., DeZeeuw, D. L., Sokolov, I., and Toth, G.: Probing the Edge of the Solar System: Formation of an Unstable Jet-Sheet, Astrophys. J., 591, L61-L65, 2003.

Opher, M., Liewer, P. C., Velli, M., Bettarini, L., Gombosi, T. I., Manchester, W., DeZeeuw, D. L., Toth, G., and Sokolov, I.: Magnetic Effects at the Edge of the Solar System: MHD Instabilities, the de Laval Nozzle Effect, and an Extended Jet, Astrophys. J., 611, 575-586, 2004.

Pogorelov, N. V.: Three-dimensional structure of the outer heliosphere in the presence of the interstellar and interplanetary magnetic fields, in: AIP Conference Proceedings 719: Physics of the outer Heliosphere: 3rd International IGPP Conference, edited by Florinski, V., Pogorelov, N. V., and Zank, G. P., 39-46, 2004.

Pogorelov, N. V. and Matsuda, T.: Influence of the interstellar magnetic field direction on the shape of the global heliopause, J. Geophys. Res., 103, 237-245, 1998.

Pogorelov, N. V. and Semenov, A. Yu.: Solar wind interaction with the magnetized interstellar medium. Shock-capturing modeling, Astron. Astrophys., 321, 330-337, 1997.

Pogorelov, N. V., Zank, G. P, and Ogino, T.: Three-dimensional Features of the Outer Heliosphere Due to Coupling between the Interstellar and Interplanetary Magnetic Fields. I. Magnetohydrodynamic Model: Interstellar Perspective, Astrophys. J., 614, 1007-1021, 2004.

Ratkiewicz, R. and Ben-Jaffel, L.: Effects of Interstellar Magnetic Field $\boldsymbol{B}$ and Constant Flux of Neutral H on the Heliosphere, J. Geophys. Res., 107, 1-13, 2002.

Ratkiewicz, R. and McKenzie, J. F.: Interstellar magnetic field effects on the termination shock, heliopause and bow shock: aligned MHD flow, J. Geophys. Res., 108, 1-8, 2003.

Ratkiewicz, R. and Webb, G. M.: On the Interaction of the Solar Wind with the Interstellar Medium: Field Aligned MHD Flow, J. Geophys. Res., 107, 1-5, 2002.

Ratkiewicz, R. and Webb, G. M.: Reply to Comment on "On the Interaction of the Solar Wind with the Interstellar Medium: Field Aligned MHD Flow by Ratkiewicz, R. and Webb, G. M.” by Pogorelov, N. V. and Matsuda, T., J. Geophys. Res., 109, 1-4, 2004.

Ratkiewicz, R., Barnes, A., Molvik, G. A., Spreiter, J. R., Stahara, S. S., Vinokur, M., and Venkateswaran, S.: Effect of varying strength and orientation of local interstellar magnetic field on configuration of exterior heliosphere: 3D MHD simulation, Astron. Astrophys., 335, 363-369, 1998.

Ratkiewicz, R., Barnes, A., and Spreiter, J. R.: Local Interstellar 
Medium and Modelling the Heliosphere, J. Geophys. Res., 105, 25 021-25 031, 2000.

Ratkiewicz, R., Barnes, A., Müller, H.-R., Zank, G. P., and Webb, G. M.: Modeling the heliosphere: Influence of the interstellar magnetic field in the presence of LISM neutral hydrogen, Ad. Sp. Res., 29, 443-438, 2002.

Ratkiewicz, R., Grygorczuk, J., and Ben-Jaffel, L.: Questions about effects of interplanetary and interstellar magnetic fields on the heliospheric interface, in: AIP Conference Proceedings 719: Physics of the outer Heliosphere: 3rd International IGPP Conference, edited by Florinski, V., Pogorelov, N. V., and Zank, G. P., 93-98, 2004.

Ratkiewicz, R., Grygorczuk, J., and Ben-Jaffel, L.: The Termination Shock and Beyond: MHD Modeling, in: AIP Conference Proceedings 781: The Physics of Collisionless Shocks: 4th International IGPP Conference, edited by Li, G., Zank, G. P., and Russell, C. T., 294-298, 2005.
Stone, E. C., Cummings, A. C., McDonald, F. b., Heikkila, B. C., Lal, N., and Webber, W. R.: Voyager 1 Explores the Termination Shock Region and the Heliosheath Beyond, Science, 309, 2017 2020, 2005.

Washimi, H. and Tanaka, T.: 3-D Magnetic Field and Current System in the Heliosphere, Space Sci. Rev., 78, 85-95, 1996.

Washimi, H. and Tanaka, T.: MHD structure of the heliosphere and its response to the 11-year solar cycle variations, Ad. Sp. Res., 23, 551-560, 1999.

Washimi, H. and Tanaka, T.: A V-shaped gutter on the nose-cone surface of the heliopause caused by MHD processes, Ad. Sp. Res., 27, 509-515, 2001.

Washimi, H. and Tanaka, T.: MHD Effects in Planetary Nebulae and in the Outer Heliosphere, in: AIP Conference Proceedings 719: Physics of the outer Heliosphere: 3rd International IGPP Conference, edited by Florinski, V., Pogorelov, N. V., and Zank, G. P., 87-92, 2004. 